\title{
Design and Realization of Intelligent Monitoring System for Soy Sauce Making Based on Embedded WEB
}

\author{
Min Zhang, a, Yu-Hua Wu ${ }^{1, b}$, Ting Yang ${ }^{1, c}$, Shi-Jun $\mathrm{Li}^{\mathrm{d} \text {, * }}$ \\ ${ }^{1}$ Jilin Agricultural University, Changchun 130118, Jilin, China \\ a1165339875@qq.com, bawuyuhua@163.com, ‘627533884@qq.com, dIsj0883@sina.com \\ *Shi-Jun LI
}

Keywords: embedded Linux, web server, koji-making, intelligent control.

\begin{abstract}
Aiming at the problem that the traditional soybean koji-making in the production process automation level is not high, the product quality and poor stability. This paper presents a new is proposed to realize the intelligent monitoring system of soybean koji-making based on embedded WEB. This project uses the S3C2440 processor and JN5148, transplanted open source Linux operating system to the S3C2440. And combined with WEB technology, the realization of temperature and humidity in the miso Quchi collection, and temperature, on the whole soybean koji-making workshop humidity, air pressure, oxygen concentration in the collection. It implements an intelligent control for miso koji-making that can improve the automatic level of the traditional soy sauce making process and guarantee the quality of the products.
\end{abstract}

\section{Introduction}

Soy sauce production need to go through steaming, koji-making, fermentation, and other aspects, which is the key link of soybean koji product quality. In the process of koji-making, it is the key factors affecting the quality of koji is temperature, humidity, concentration of oxygen [1-3]. But the traditional soybean koji production in the process of temperature, humidity, oxygen concentration, pressure completely by skilled workers with personal experience of regulation. Although the traditional miso manufacturing process has good taste, unique flavor, high nutritional value, but it is difficult to achieve the best quality, production is also lower than industry standards, and the stability of the product is poor.

Based on the advantages and characteristics of the traditional miso manufacturing process, improving the product quality and maintaining the stability of the product, combining the advanced automation technology with the communication technology, real-time monitoring the process of making the miso to achieve the optimized management and reliable operation of the purpose. At present, embedded environment monitoring is used, such as environmental monitoring of vegetable greenhouses [4-5], grain conditions [6-7].

This paper presents an intelligent monitoring system of soy sauce making based on WEB. This project uses the S3C2440 processor and JN5148, transplanted open source Linux operating system to the S3C2440. And combined with WEB technology, which can display the environmental parameters in the curved pool and the environmental parameters in the koji workshop. The system can control the turning machine, blower and ventilation gate of the koji workshop in time to adjust the environmental parameters of the koji and koji workshop.

\section{Overall System Framework}

The whole system is mainly composed of embedded field acquisition module and monitoring server. The overall system framework is shown in Fig.1. Field information collection module and the implementing agencies and S3C2440 monitoring server connection, the use of JN5148 terminal nodes will be transmitted to the coordinator through the serial port transmission and storage to the S3C2440 processor. The user communicates with the service center through the Internet network access, through the browser on soybean koji environmental parameters monitoring of browsing and 
querying, so on realize the real-time monitoring and management of koji workshop equipment and environmental parameters.

Field acquisition module equipment is produced by the JN5148 company Jennic chip, using ZigBee protocol to collect the temperature and humidity for wireless transmission. It transplanted open source Linux operating system to the S3C2440, which the external integrated DM9000 network card chip, USB interface and serial port, to achieve the information and data acquisition workshop karaoke network transmission.

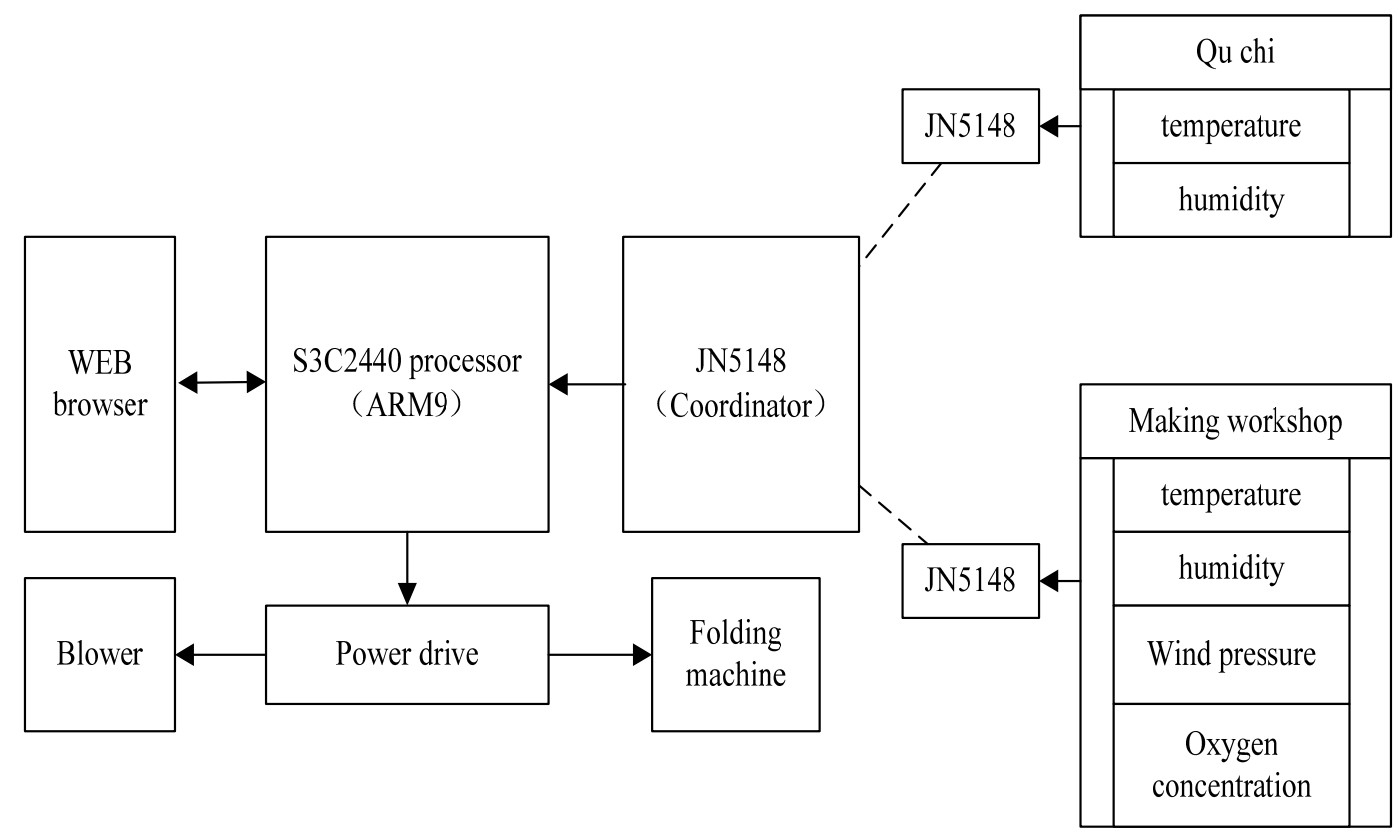

Fig. 1 The overall system framework

\section{System Software Design}

The software design includes the transplantation of U-Boot, the transplantation of Linux system, the realization of function module and the realization of ZigBee. As Linux itself has source code development, the kernel can cut, take up less memory and more technical support and other features, with the embedded Linux technology continue to mature, its customization more convenient, while supporting more platforms.

\subsection{Transplantation of Embedded System}

The embedded Linux operating system of the building is divided into five parts, namely the establishment of cross compiler environment, the transplantation of U-Boot, according to the hardware platform for the reduction of the Linux kernel and driver module corresponding to the construction and development of the user root file system program.

In the host platform to install the corresponding cross-compiler tool chain arm-linux-gcc-4.3.2.tgz. Through the Bootloader, you can initialize the hardware device, the establishment of the system memory space mapping, the system software and hardware to the appropriate state, to call the operating system kernel to prepare the correct environment. The system used U-Boot-2012.04.01. for U-Boot. Operating system kernel for Linux-2.6.22.6, enter the directory to extract, configure and compile.

File system is an important part of embedded operating system, which is the operating system responsible for the management and storage of file information software organizations. Download busybox-1.7.0 production root file system. Linux system supports multiple file systems, the system uses Yaffs2 file system. Yaffs2 comes with NAND chip driver, and provides the embedded system 
with direct access to the file system API, the user can not use the MTD and VFS in Linux, the file directly to the operation.

\subsection{The Construction of Embedded WEB Server}

Embedded WEB server's main function is to monitor the client's service request, according to the customer's request type, return to the static page or the corresponding CGI application as a response.

Boa server in the arm platform migration process:

1) Compile the boa program. Download boa-0.94.13.tar.gz and extract the source code, execute. / Configure, and then modify the Makefile, set the compiler to arm-linux-, the implementation of make. In order to reduce the size of boa, save space can be removed debugging part of the information, then execute arm-linux-strip boa.

2) Modify the configuration file boa.conf. Create /etc/boa directory, and copy boa.conf to the directory, modify the permissions of boa.conf, set the error log directory Errorlog and access log directory AccessLog. The two parts of the log must be writable, or an error occurs. Set the html file directory, the default home page and CGI script directory.

3) Test by http://192.168.194.128 (IP ARM address) access in Windows. The test interface is shown in Fig.2.

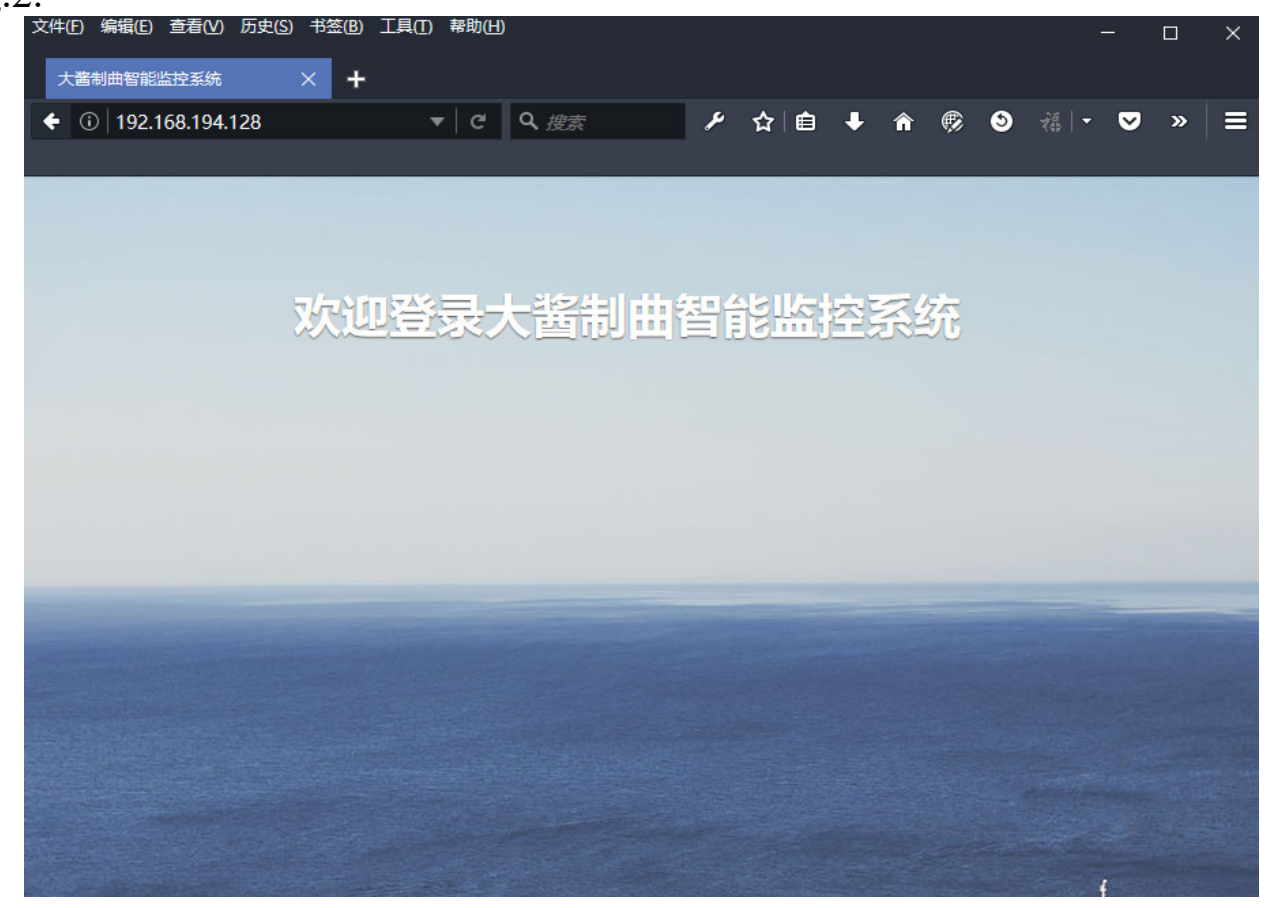

Fig. 2 The test interface

\subsection{The Design of CGI Program}

The present development of Web method to generate dynamic Web pages with CGI and server script, such as PHP, ASP and so on, but because it is embedded in the Web server, taking into account the embedded server resources are limited, usually provide CGI support, so in the embedded equipment Web application is based on the development of CGI.

CGI (Common Gate Interface) is a section of running on the Web server program, which provides the interface with the client Html page [8]. After building a WebBoa, the user input access to the IP address on the computer browser that can be remote login monitoring system's home page. The user login interface is shown in Fig.3. 


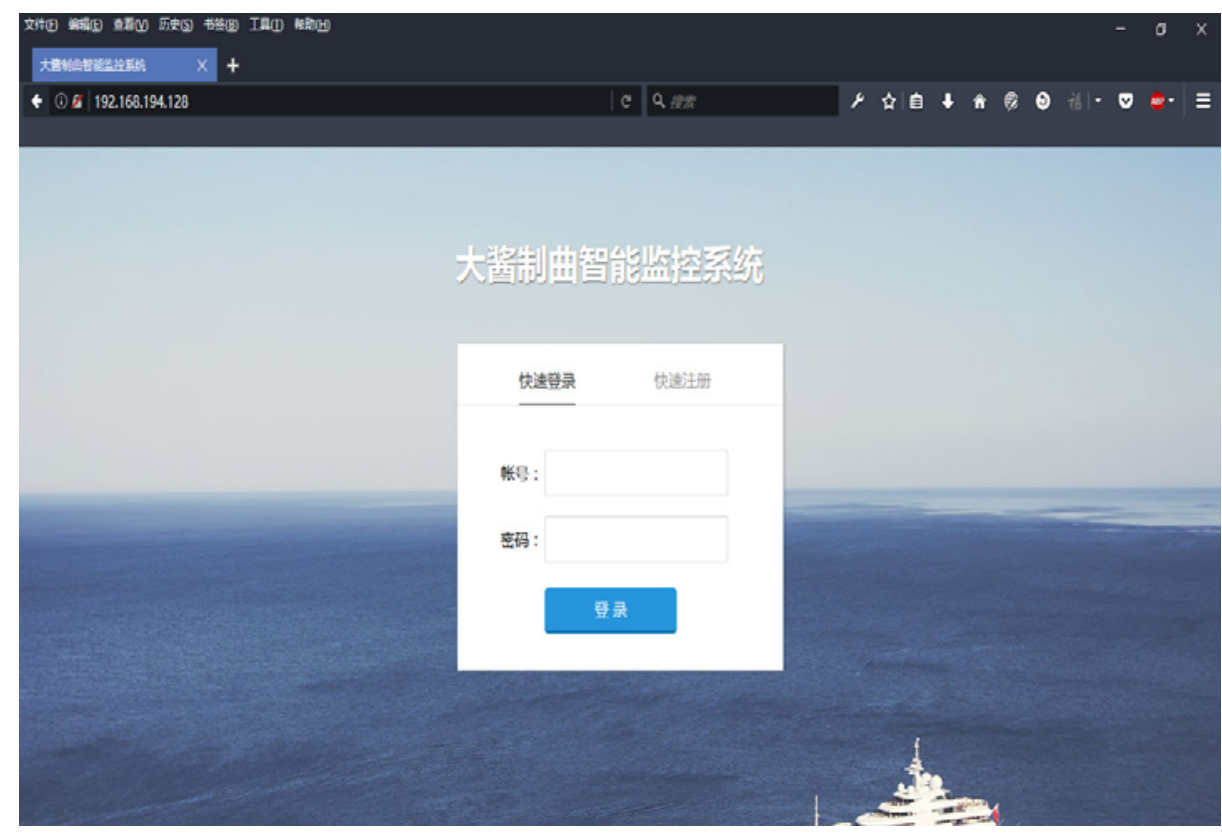

Fig. 3 The user login interface

\section{Communication Protocol Design}

ZigBee [9] technology is a low-rate, low-power, low-cost wireless network solution for automation and wireless control. ZigBee networking includes Coordinator, Router and EndDevice. The coordinator is responsible for the formation, maintenance and control of the joining of the terminal nodes; the router is responsible for packet routing; the terminal node is responsible for data collection.

In this system, the JN5148 module component network is adopted. The sensor data is transferred to the coordinator through the ZigBee node and then transmitted to the ARM processor for data processing. The ZigBee wireless network protocol model is shown in Fig.4.

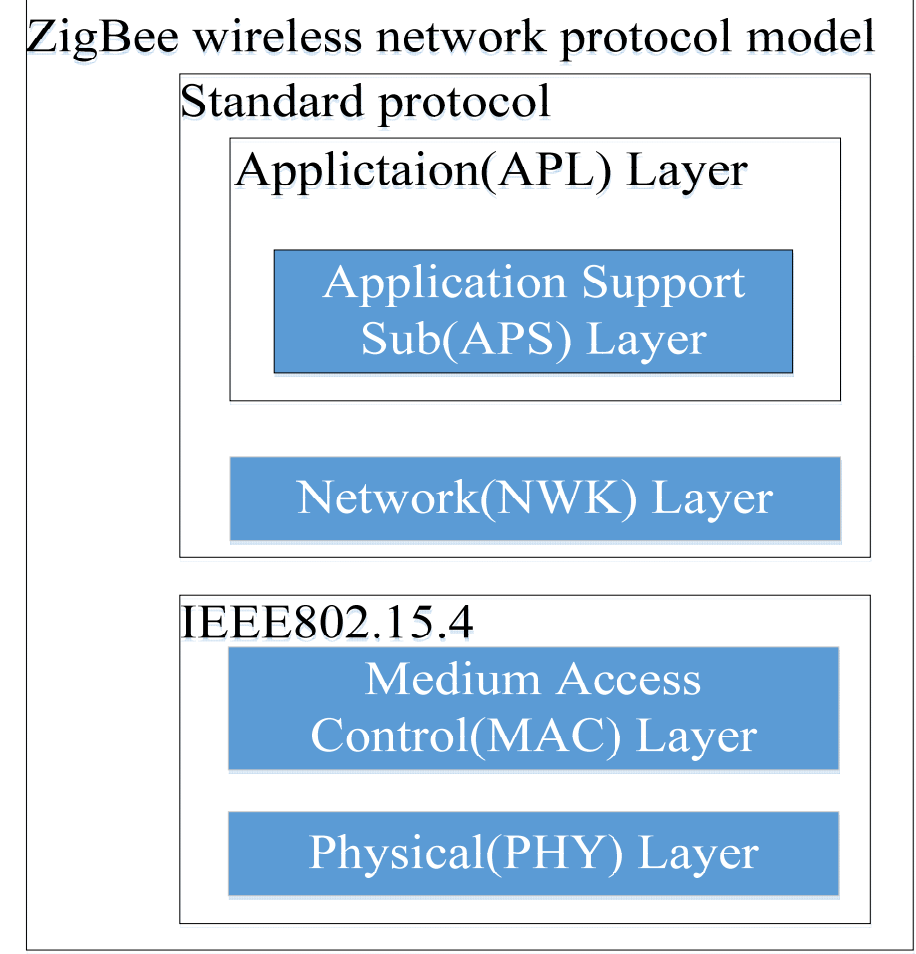

Fig. 4 The ZigBee wireless network protocol model 


\section{Conclusions}

This paper combines ARM-Linux and ZigBee protocol, using Web programming (CGI) as the core technology, the preparation of the hardware driver, the realization of temperature and humidity in the miso Quchi collection, and temperature, on the whole, Soybean Koji workshop humidity, air pressure, the oxygen concentration in the collection. That can improve the automatic level of the traditional soy sauce making process and guarantee the quality of the products

\section{Acknowledgments}

The authors wish to express their gratitude to the projects: Design and Realization of Intelligent Monitoring System for Soy Sauce Making Based on Embedded WEB from Education Department of Jilin Province, Jilin Province Economic Structural Adjustment Leading Fund Special Project (No. 2014Y108) and Changchun City Science and Technology Plan Project (No. 14nk029), Jilin Province Science and Technology Development Program Funded Project (No. 20160623016TC), Education Department of Jilin Province's $12^{\text {th }}$ Five-Year Science and Technology Research Project (2015175), for their generous support of this work. At the same time, the other authors also thank the corresponding author, Shijun LI, for his assistance in submitting this paper.

\section{References}

[1] LIN Zushen. Change of enzyme activity in Aspergillus oryzae cultivation and technical optimization[J]. Chinese Brewing, 2007,05:56-59, in Chinese.

[2] ZHANG Yubin, YANG Li. The soy sauce is researched into process of making flexure[J]. Chinese Condiment, 2004(12):20-22, in Chinese.

[3] Tan Wufeng, Li Yuelan, Xu Fuyou. Countermeasure and Reason Causing Ammonia Smell in the Making-leaven of the Soy[J]. Guangzhou food industry science and technology, 2003(4):46-47, in Chinese.

[4] SHANG Hai, GUO Haiyan, XIA Guiping, WU Jingyue, XIA Zhi. Temperature Monitoring and Alarming System for Greenhouse Based on LabVIEW[J]. Journal of Zhejiang Agricultural Sciences, 2014(11):1777 1779, in Chinese.

[5] YU Huafang et al. Temperature and Humidity Control System in Vegetables Greenhouse[J]. Journal of Anhui Agricultural Sciences, 2011,39(28):17601-17603, in Chinese.

[6] Jiang Hui;Zhen Tong;Wang Feng. Research and design of the remote grain monitoring system based on ARM/ZigBee[J]. Journal of Chinese Agricultural Mechanization, 2015,02:99-103+98, in Chinese.

[7] WANG Shuai, Based On S3C2440A Design Of Temperature And Humidity Measurement And Control System[D]. Wuhan polytechnic university, 2015, in Chinese.

[8] WANG Guowei1, SONG Tiecheng1, CHEN Zhengshi2. Video Surveillance Server Based on Embedded Web Server[J]. Computer Engineering, 2005,31(22):202-204, in Chinese.

[9] ANG Zhiin, JIN Haihong, FAN Zhiguo, et al. WSN node desian and communication realization based on ZigBee protocol[J]. Modern Electronics Technique, 2007, 30(10):47-49, in Chinese. 